\title{
Acute liver failure in the setting of herpes simplex virus and coexistent acute fatty liver of pregnancy
}

\author{
Julius Wilder ${ }^{1,2}$, Sydney Chang ${ }^{3}$, Diana Cardona ${ }^{4}$, Keyur Patel ${ }^{1,2}$, Carla Brady ${ }^{1}$ \\ 1. Division of Gastroenterology, Duke University School of Medicine, United States. 2. Duke Clinical Research Institute, \\ United States. 3. Department of Obstetrics and Gynecology, Albert Einstein College of Medicine, United States. 4. Duke \\ Department of Pathology, Duke University School of Medicine, United States.
}

Correspondence: Carla Brady. Address: DUMC 3913, Durham, NC, United States. E-mail: carla.brady@dm.duke.edu

Received: November 21, 2014

Accepted: February 8, 2015

Online Published: February 13, 2015

DOI : $10.5430 /$ crcp.v2n2p89

URL: http://dx.doi.org/10.5430/crcp.v2n2p89

\begin{abstract}
Fulminant hepatitis is a rare complication of herpes simplex virus (HSV-1 and HSV-2). Another rare cause of fulminant hepatitis in pregnant women is acute fatty liver of pregnancy (AFLP). Here we present a female with fulminant hepatitis after a cesarean section whose infant clinically decompensated in the early neonatal period. Mother and child were diagnosed with fulminant hepatic failure from HSV, and the mother was found to have coexistent fatty liver of pregnancy on biopsy. Thus, two rare causes of fulminant hepatitis were co-morbid in the same patient. Rapid diagnosis enabled successful treatment, and both mother and infant recovered well.
\end{abstract}

\section{Key words}

Hepatology, Liver failure, Fulminant hepatitis, Herpes simplex virus, Acute fatty liver of pregnancy

\section{Case report}

The patient is a 23 years old G1P1001 whose only medical history consisted of asthma, degenerative disk disease, depression/anxiety, and fibromyalgia. She had no history of recent travel or high-risk behaviors such as alcohol or illicit drug use. She was married with a history of one lifetime sexual partner. After an uneventful pregnancy, she developed intermittent fevers for one week prior to her delivery. There was concern for rupture of membranes, and she was admitted for augmentation of labor. Throughout her labor course, she had persistent fevers and ultimately underwent a cesarean section for suspected chorioamnionitis at 41 weeks and 0 days.

On postpartum day 3, the infant developed increasing respiratory distress, lethargy, poor feeding, and decreased urine output. The infant continued to decline clinically, requiring admission to the neonatal intensive care unit and use of an oscillator for respiratory support. The infant developed acute hepatic decompensation with jaundice, thrombocytopenia, and AST and ALT levels that were 50-100 times the upper limit of normal ULN.

Following delivery, the patient continued to have intermittent fevers that eventually resolved with antibiotics. Although the infant continued to require critical care, the patient was discharged to home after being afebrile for 36 hours. She returned to the hospital on post-partum day 6 with recurrence of fevers, altered mental status, and abdominal pain. On admission, her physical examination showed a blood pressure of 107/57, a pulse of 95, respirations of 20/min, an oxygen 
saturation of $95 \%$, and a temperature of $36.0^{\circ} \mathrm{C}$. Her heart and lung sounds were normal. Her abdominal exam revealed diffuse tenderness to palpation with no rebound or guarding. The surgical scar from her C-section was without erythema and induration. Although she was initially alert and oriented, she subsequently exhibited intermittent episodes of severe confusion.

The laboratory assessment was remarkable for thrombocytopenia with a platelet count of $52 \times 10^{9} / \mathrm{L}$, and her leukocyte count was $3.5 \times 10^{9} / \mathrm{L}$. She exhibited evidence of acute kidney injury with a creatinine of $1.5 \mathrm{mg} / \mathrm{dL}$. Amylase and lipase values were within normal limits. Her transaminases were elevated with an ALT of 1,307 IU/L and AST of 3,935 IU/L. She also exhibited a cholestatic pattern of liver injury with an elevated alkaline phosphatase of 549 and a total bilirubin level of $4.0 \mu \mathrm{mol} / \mathrm{L}$ ( $2.2 \mu \mathrm{mol} / \mathrm{L}$ conjugated). The INR was elevated at 1.9 (PT 22.6 seconds).

An ultrasound of her liver with doppler measurement was obtained and was remarkable for a heterogeneous echotexture of the liver as well as blood flow in the normal direction in the major hepatic vessels. A CT of her abdomen with contrast revealed ascites, hepatomegaly, and diffuse heterogeneous enhancement of the liver with numerous nodular, hypoenhancing foci that were more confluent in the left hepatic lobe (see Figure 1).

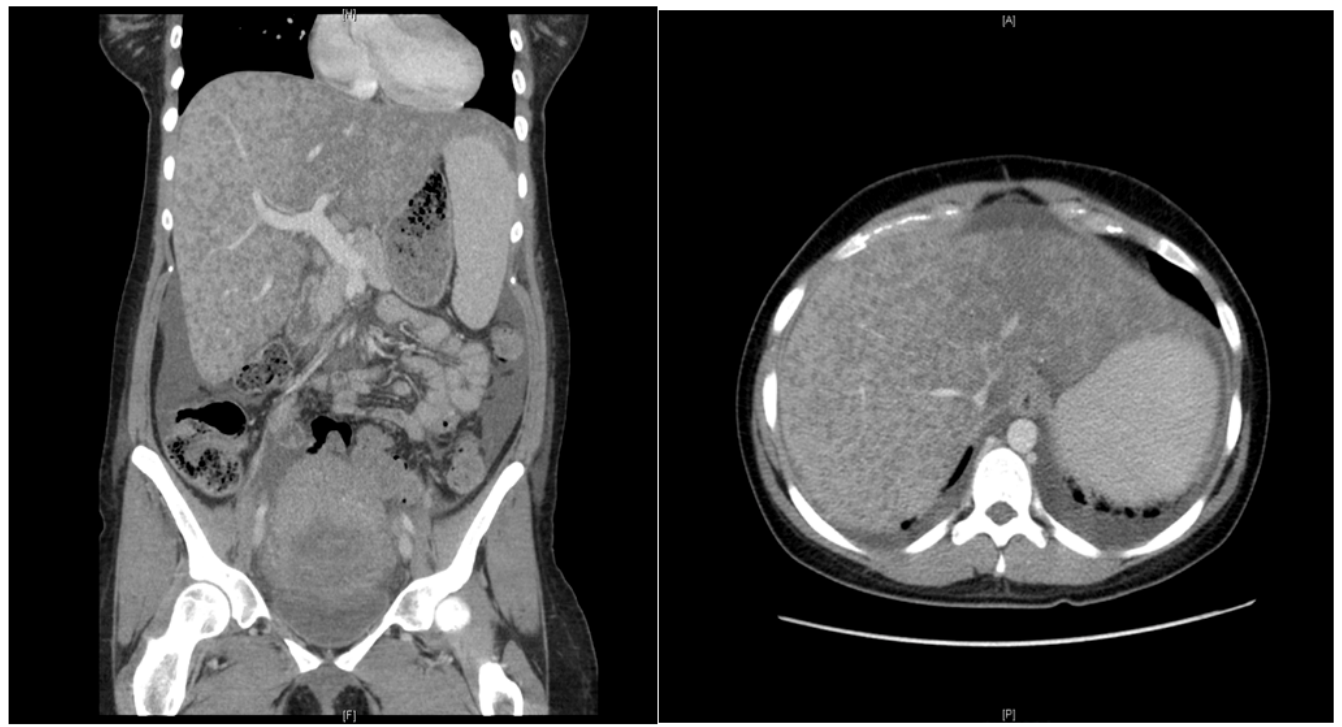

Figure 1. CT Abdomen and pelvis

As shown in Figure 1, the CT abdomen with contrast shows ascites, hepatomegaly, and diffuse heterogeneous enhancement of the liver with numerous nodular hypoenhancing foci that is more confluent in the left hepatic lobe.

Over the next few hours, the patient developed worsening encephalopathy and was transferred to the surgical intensive care unit and intubated. At that time, her clinical presentation was believed to favor acute fatty liver of pregnancy (AFLP), although the significant transaminitis was concerning for viral or toxin-mediated injury. While awaiting an urgent transjugular liver biopsy, the surgical ICU team was notified that the infant's serology was positive for herpes simplex virus (HSV-2). The mother was immediately started on IV acyclovir therapy before her results of her HSV and other viral serology became available. A liver biopsy obtained a few hours later showed submassive hepatic necrosis involving approximately $75 \%$ of the biopsy tissue. Within the areas of necrosis, dying hepatocytes demonstrated smudgy nuclei with margination of the chromatin consistent with herpes infection. Immunohistochemical studies for HSV revealed strong immunoreactivity within hepatocytes. Additionally, electron microscopy revealed intranuclear enveloped viral particles consistent with HSV (see Figure 2A-B). Her biopsy was also remarkable for patchy centrilobular microvesicular steatosis in areas devoid of necrosis, suggestive of a coexistent AFLP (see Figure 2C-D). Ascitic fluid from subsequent paracentesis and blood serum were positive for HSV-2. 


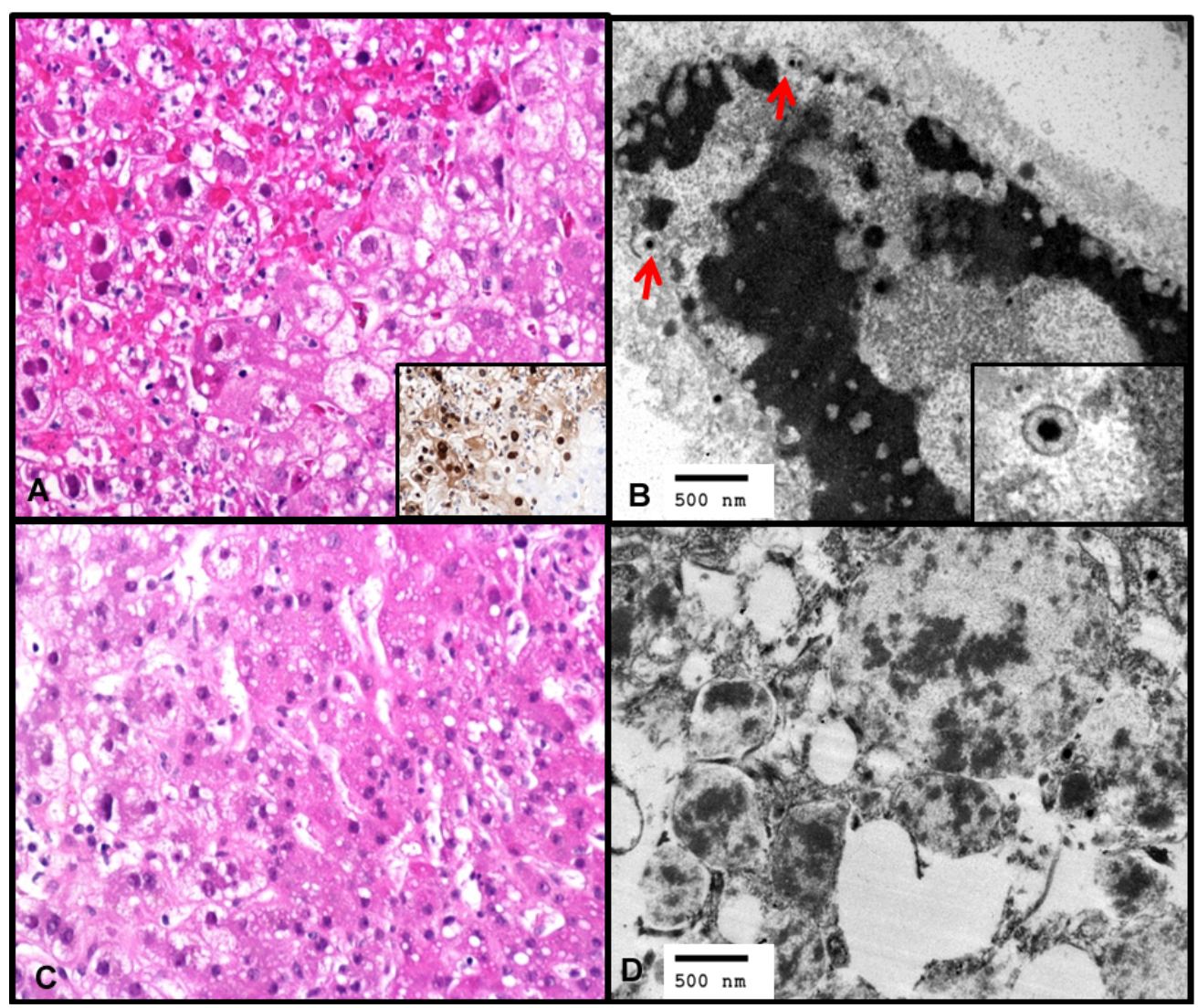

Figure 2. Liver biopsy. (A) Area of hepatocyte necrosis with classic viral cytopathic effect for HSV, in the form of smudgy nuclei, margination of the chromation and focal multinucleation (H\&E, 200×). Inset, immunohistochemistry for HSV reveal positive nuclear and cytoplasmic expression. (B) Electron microscopy revealed the presence of intranuclear viral particles composed of dense cores and surrounding capsid, consistent with HSV. (C) In areas away from necrosis and involvement by HSV, microvesicular and small droplet macrovesicular steatosis was identified (H\&E, 200×). (D) Electron microscopic examination also revealed the presence of microvesicular steatosis.

Following initiation of treatment with acyclovir, the patient had marked improvement in her transaminitis. Although her AST and ALT levels peaked at 4,295 U/L and 1,307 U/L respectively, her transaminases decreased significantly within the first week of treatment (see Figure 3). While both her ascitic fluid and serum were positive for HSV, the mother never displayed any mucocutaneous herpetic lesions. Both the infant and patient made a full recovery. The patient was weaned from the ventilator and extubated after six days. She was then transferred to a general medical floor bed where her recovery continued.

Figure 3. Timeline of AST and ALT trend

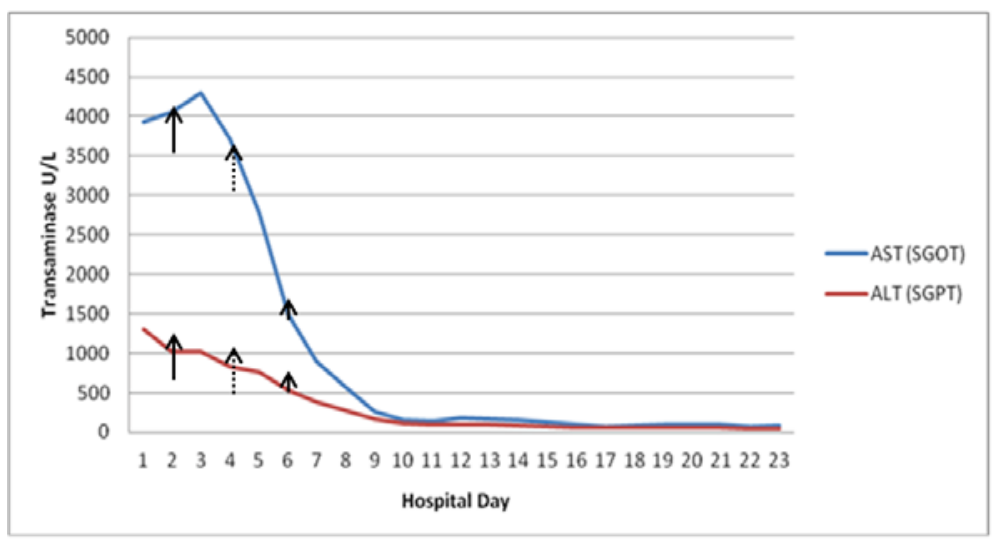


As shown in Figure 3, timeline of AST and ALT Trend following Acyclovir Initiation. Solid arrow (initiation of acyclovir therapy), dashed arrow (biopsy diagnosis), and arrow head (+HSV serum PCR).

\section{Discussion}

HSV infection is endemic with an estimated $80 \%$ of adults likely to contract HSV throughout their lifetime ${ }^{[1]}$. Although HSV infection may present with mild nonspecific viral symptoms, most HSV infections are asymptomatic. Only $30 \%-50 \%$ show characteristic herpetic skin lesions ${ }^{[2-4]}$. Fulminant HSV hepatitis is a rare presentation of HSV infection and accounts for only $1 \%$ of all acute liver failure cases and $2 \%$ of all viral causes of acute liver failure 4 . While the vast majority of those infected with HSV are immunocompromised, up to $25 \%$ of patients who develop HSV hepatitis are immunocompetent ${ }^{[3]}$.

Twenty seven cases of HSV hepatitis during pregnancy have been reported to date ${ }^{[5]}$. The diagnosis of disseminated HSV hepatitis can be difficult in pregnancy, and as with our case, most cases of disseminated HSV hepatitis do not have typical mucocutaneous lesions. Commonly seen clinical features of HSV hepatitis include fever (82\%), anorexia with nausea and/or vomiting (18\%), abdominal pain (33\%), leukopenia (43\%), and coagulopathy (20\%) ${ }^{[6]}$. The typical oral and/or genital lesions seen in HSV occur in 30\% of patients with HSV hepatitis. Disseminated extrahepatic involvement in the lungs, lymph nodes, spleen, or adrenal glands can occur. The liver profile can be helpful in the detection of HSV hepatitis. The majority of patients with HSV hepatitis (as high as 90\%) have a characteristic liver profile described as "anicteric hepatitis" ${ }^{[5,7]}$. Anicteric hepatitis is a pattern of liver injury in which a significant increase in transaminases $(100-1,000$ fold) occurs in the setting of relatively normal or low bilirubin levels ${ }^{[1,3,5,7,8]}$. This pattern is often associated with a marked elevation of AST greater than ALT. Diagnosis of HSV hepatitis may include liver biopsy or detection of viremia. However, evaluation for HSV via viral PCR testing is not always available and takes time. The gold standard of diagnosis is liver biopsy. Described as nuclei with large eosinophilic ground glass-like inclusions surrounded by a clear halo, Cowdry Type A inclusions are pathognomonic for HSV hepatitis. However, margination of the chromatin, multinucleation and nuclear molding are more commonly seen ${ }^{[9-11]}$. Immunohistochemical staining can confirm the diagnosis of HSV.

Acute liver failure develops in $74 \%$ of patients with HSV hepatitis. Of these cases, the mortality rate approaches $90 \%{ }^{[1,3,5,8]}$. Data support the use of acyclovir to treat HSV hepatitis, and early initiation of treatment is vital to transplantfree survival ${ }^{[7]}$. Hence, acyclovir is reasonable empiric therapy for acute liver failure of unknown etiology.

Our patient presented with both atypical and typical clinical signs of HSV hepatitis. She had fever, abdominal pain, relative leukopenia, and coagulopathy. Although her ascitic fluid and serum were positive for HSV-2, she displayed no mucocutaneous lesions consistent with HSV. Viremia and disseminated infection occur more in HSV-2 and in primary infection. HSV-1 antibodies were not obtained. Although there is no mention of multi-nucleated giant cells within the liver biopsy, electron microscopy revealed intranuclear and cytoplasmic viral particles composed of dense cores with surrounding capsids and positive immunohistochemistry for HSV. Her laboratory pattern of liver injury showed a significant transaminitis with her AST markedly higher than the ALT. However, she also had a significant elevation of her total bilirubin level; hence, the classic anicteric pattern of liver injury was not present. Part of the rise in her total bilirubin level may be explained by the presence of underlying acute fatty liver of pregnancy.

AFLP is a rare and potentially fatal complication of pregnancy that typically occurs in the third trimester or in the immediate postpartum period ${ }^{[6]}$. The incidence of AFLP is approximately one in 10,000 to 15,000 pregnancies ${ }^{[6]}$. It tends to occur more often in the setting of multiple gestations and in women who are underweight. Maternal and perinatal mortality have been reported as high as $75 \%$ and $85 \%$ respectively. However, with prompt diagnosis and appropriate treatment, maternal and perinatal mortality may decrease to approximately $18 \%$ and $23 \%$, respectively ${ }^{[6]}$. 
Early diagnosis of AFLP is difficult because of the varying degrees of severity of symptoms and similarity of symptoms seen in the third trimester of a normal pregnancy. The most frequent initial symptoms are nausea or vomiting (approximately 75\% of patients) and abdominal pain (particularly epigastric, 50\%). Up to one-half of patients have signs of preeclampsia at presentation or develop preeclampsia during the course of illness ${ }^{[12]}$. Hemolysis, elevated liver enzymes and low platelets (HELLP) syndrome, pre-eclampsia, thrombotic thrombocytopenia purpura and AFLP may all be a spectrum of the same illness. Extrahepatic complications include infection, intra-abdominal bleeding, central diabetes insipidus, and pancreatitis ${ }^{[13-15]}$. Laboratory abnormalities typically include serum aminotransferase elevations in the range of $1,000 \mathrm{IU} / \mathrm{L}$. Serum bilirubin levels are also usually elevated, and the white blood cell count is typically greater than $15 \times 10^{9} / \mathrm{L}^{[16]}$. The platelet count may be decreased with or without other signs of disseminated intravascular coagulation ${ }^{[17]}$. Elevations in serum ammonia levels, prolongation of prothrombin time, hypoglycemia, and acute renal failure are often present in severe cases ${ }^{[18]}$. Our patient presented with clinical symptoms, relative thrombocytopenia and coagulopathy that could be attributed to AFLP. However, her relative leukopenia, febrile illness and high transaminitis were more typical of viral or toxin mediated injury.

Long chain 3-hydroxyacyl-coenzyme A dehydrogenase deficiency, an inherited enzyme deficiency in beta-oxidation, may predispose certain women to AFLP ${ }^{[19]}$. AFLP is typically a clinical diagnosis, and HELLP syndrome must be excluded given the clinical overlap between the two diseases. Evidence of hepatic insufficiency (hypoglycemia and encephalopathy) is suggestive of AFLP. Imaging is not diagnostic, and evidence of fat on ultrasound or computed tomography is most useful in retrospect several months following the peak of the illness. In the context of the appropriate clinical presentation, liver biopsy is diagnostic of AFLP, revealing the presence of microvesicular steatosis. This fatty infiltration is most prominent in central and mid-zonal parts of the lobule and usually spares a sharply defined rim of cells around the portal tracts The hepatocytes demonstrate centrally located nuclei that are surrounded by small fat droplets, which give the cytoplasm a foamy appearance ${ }^{[20]}$. Treatment of AFLP typically consists of prompt and often emergent delivery and conservative measures to stabilize and support the mother. AFLP can recur in subsequent pregnancies, but the exact risk of recurrence is unknown. Although most severely ill patients recover and have no sequelae of the liver disease itself, substantial morbidity and mortality can occur ${ }^{[21]}$. Death from AFLP is usually secondary to sepsis, renal failure, circulatory collapse, pancreatitis or gastrointestinal bleeding ${ }^{[6,16,22]}$.

\section{Conclusions}

Both HSV hepatitis and AFLP are rare causes of morbidity and mortality. We report a rare case of fulminant hepatitis as a result of coexisting HSV infection and AFLP. We are able to identify only one previously published case report of concurrent HSV hepatitis and AFLP ${ }^{[23]}$. Our patient had classic histologic and radiographic findings seen in AFLP and HSV hepatitis. While liver biopsy was awaited, the patient received prompt treatment with acyclovir largely because her newborn baby's HSV PCR was positive, and liver pathology confirmed the diagnosis 48 hours before the mother's serum HSV PCR results were available. Given the high mortality rate of fulminant HSV hepatitis, maintaining a high clinical index of suspicion, obtaining early histopathological confirmation and institution of empiric treatment with acyclovir should be considered in all populations, including pregnant women, who are at risk and present with acute liver failure of unknown etiology.

\section{Funding and acknowledgements}

While no funding was directed to obtaining the supply of data, J.W. was supported by the Duke NIDDK Grant No. 5T32-DK7568-22; KP -Consultant and/or Grant/Research support from Gilead Sciences, Santaris, BMS, Benitec, and Merck. None of the other authors have any conflicts of interest. 


\section{References}

[1] Riediger C, Sauer P, Matevossian E, et al. Herpes simplex virus sepsis and acute liver failure. Clin Transplant. Dec 2009; 23 Suppl 21: 37-41. PMid: 19930315. http://dx.doi.org/10.1111/j.1399-0012.2009.01108.x

[2] Schiodt FV, Davern TJ, Shakil AO, et al. Viral hepatitis-related acute liver failure. Am J Gastroenterol. Feb 2003; 98(2): $448-453$. http://dx.doi.org/10.1016/S0002-9270(02)05891-4

[3] Norvell JP, Blei AT, Jovanovic BD, et al. Herpes simplex virus hepatitis: an analysis of the published literature and institutional cases. Liver Transpl. Oct 2007; 13(10): 1428-1434. PMid: 17902129. http://dx.doi.org/10.1002/lt.21250

[4] Farr RW, Short S, Weissman D. Fulminant hepatitis during herpes simplex virus infection in apparently immunocompetent adults: report of two cases and review of the literature. Clin Infect Dis. Jun 1997; 24(6): 1191-1194. PMid: 9195081. http://dx.doi.org/10.1086/513646

[5] Allen RH, Tuomala RE. Herpes simplex virus hepatitis causing acute liver dysfunction and thrombocytopenia in pregnancy. Obstet Gynecol. Nov 2005; 106(5 Pt 2): 1187-1189. PMid: 16260566. http://dx.doi.org/10.1097/01.AOG.0000160481.11517.a0

[6] Ko H, Yoshida EM. Acute fatty liver of pregnancy. Can J Gastroenterol. Jan 2006; 20(1): 25-30. PMid: 16432556.

[7] Peters DJ, Greene WH, Ruggiero F, et al. Herpes simplex-induced fulminant hepatitis in adults: a call for empiric therapy. Dig Dis Sci. Dec 2000; 45(12): 2399-2404. PMid: 11258565. http://dx.doi.org/10.1023/A:1005699210816

[8] Goyert GL, Bottoms SF, Sokol RJ. Anicteric presentation of fatal herpetic hepatitis in pregnancy. Obstet Gynecol. Apr 1985; 65(4): 585-588. PMid: 4039045.

[9] Abbo L, Alcaide ML, Pano JR, et al. Fulminant hepatitis from herpes simplex virus type 2 in an immunocompetent adult. Transpl Infect Dis. Dec 2007; 9(4): 323-326. PMid: 17605748. http://dx.doi.org/10.1111/j.1399-3062.2007.00207.x

[10] Pinna AD, Rakela J, Demetris AJ, et al. Five cases of fulminant hepatitis due to herpes simplex virus in adults. Dig Dis Sci. Apr 2002; 47(4): 750-754. PMid: 11991604. http://dx.doi.org/10.1023/A:1014779614525

[11] Sharma S, Mosunjac M. Herpes simplex hepatitis in adults: a search for muco-cutaneous clues. J Clin Gastroenterol. Sep 2004; 38(8): 697-704. PMid: 15319655. http://dx.doi.org/10.1097/01.mcg.0000135365.20418.b8

[12] Knox TA, Olans LB. Liver disease in pregnancy. N Engl J Med. Aug 22 1996; 335(8): 569-576. PMid: 8678935. http://dx.doi.org/10.1056/NEJM199608223350807

[13] Riely CA. Acute fatty liver of pregnancy. Semin Liver Dis. Feb 1987; 7(1): 47-54. PMid: 3296215. http://dx.doi.org/10.1055/s-2008-1040563

[14] Pereira SP, O'Donohue J, Wendon J, et al. Maternal and perinatal outcome in severe pregnancy-related liver disease. Hepatology. Nov 1997; 26(5): 1258-1262. PMid: 9362370.

[15] Kennedy S, Hall PM, Seymour AE, et al. Transient diabetes insipidus and acute fatty liver of pregnancy. Br J Obstet Gynaecol. May 1994; 101(5): 387-391. PMid: 8018608. http://dx.doi.org/10.1111/j.1471-0528.1994.tb11909.x

[16] Moldenhauer JS, O'Brien J M, Barton JR, et al. Acute fatty liver of pregnancy associated with pancreatitis: a life-threatening complication. Am J Obstet Gynecol. Feb 2004; 190(2): 502-505. PMid: 14981397. http://dx.doi.org/10.1016/j.ajog.2003.09.022

[17] Usta IM, Barton JR, Amon EA, et al. Acute fatty liver of pregnancy: an experience in the diagnosis and management of fourteen cases. Am J Obstet Gynecol. Nov 1994; 171(5): 1342-1347. http://dx.doi.org/10.1016/0002-9378(94)90158-9

[18] Castro MA, Goodwin TM, Shaw KJ, et al. Disseminated intravascular coagulation and antithrombin III depression in acute fatty liver of pregnancy. Am J Obstet Gynecol. Jan 1996; 174(1 Pt 1): 211-216. http://dx.doi.org/10.1016/S0002-9378(96)70396-4

[19] Treem WR, Shoup ME, Hale DE, et al. Acute fatty liver of pregnancy, hemolysis, elevated liver enzymes, and low platelets syndrome, and long chain 3-hydroxyacyl-coenzyme A dehydrogenase deficiency. Am J Gastroenterol. Nov 1996; 91(11): 2293-2300. PMid: 8931405.

[20] Bacq Y. Acute fatty liver of pregnancy. Semin Perinatol. Apr 1998; 22(2): 134-140. http://dx.doi.org/10.1016/S0146-0005(98)80045-1

[21] Castro MA, Fassett MJ, Reynolds TB, et al. Reversible peripartum liver failure: a new perspective on the diagnosis, treatment, and cause of acute fatty liver of pregnancy, based on 28 consecutive cases. Am J Obstet Gynecol. Aug 1999; 181(2): $389-395$. http://dx.doi.org/10.1016/S0002-9378(99)70567-3

[22] Ranjan V, Smith NC. Acute fatty liver of pregnancy. J Obstet Gynaecol. May 1997; 17(3): 285-286. PMid: 15511855. http://dx.doi.org/10.1080/01443619750113320

[23] Luzar B, Ferlan-Marolt V, Poljak M, et al. Acute fatty liver of pregnancy -- an underlying condition for herpes simplex type 2 fulminant hepatitis necessitating liver transplantation. Z Gastroenterol. May 2005; 43(5): 451-454. PMid: 15871067. http://dx.doi.org/10.1055/s-2005-857952 